\title{
Calpain7 Negatively Regulates Human Endometrial Stromal Cell Decidualization in Endometriosis by Promoting Fox01's Phosphorylation and Nuclear Exclusion via Hydrolyzing AKT1
}

\section{Nannan Kang}

Reproductive Medicine Center: University of Minnesota Physicians

\section{Huizhi Shan}

Reproductive Medicin Center, The affiliated Drum Tower Hospital of Nanjing University Junxia Wang

Reproductive Medicine Center, The affiliated Drum Tower Hospital of Nanjing University Jie Mei

Reproductive Medicine Center, The affiliated Drum Tower Hospital of Nanjing University Yue Jiang

Reproductive Center, The affiliated Drum Tower Hospital of Nanjing University

Jidong Zhou

Reproductive Medicine Center, The affiliated Drum Tower Hospital of Nanjing University

\section{Chenyang Huang}

Reproductive Medicine Center, The affiliated Drum Tower Hospital of Nanjing University

\section{Hui Zhang}

Reproductive Medicine Center, The affiliated Drum Tower Hospital of Nanjing University

\section{Mei Zhang}

Reproductive Medicine Center, The affiliated Drum Tower Hospital of Nanjing University

\section{Xin Zhen}

Reproductive Medicine Center, The affiliated Drum Tower Hospital of Nanjing University

\section{Guijun Yan ( $\nabla$ yanguijun55@163.com )}

Reproductive Medicine Center, The affiliated Drum Tower Hospital of Nanjing University https://orcid.org/0000-0001-9628-1211

\section{Haixiang Sun}

Reproductive Medicine Center, The affiliated Drum Tower Hospital of Nanjing University

\section{Research}

Keywords: CAPN7, Fox01, AKT1, decidualization EMs 
Posted Date: November 1st, 2021

DOl: https://doi.org/10.21203/rs.3.rs-985926/v1

License: (c) (i) This work is licensed under a Creative Commons Attribution 4.0 International License. Read Full License 


\section{Abstract \\ Background}

Endometrial receptivity damage caused by impaired decidualization may be one of the mechanisms of infertility in endometriosis (EMs). Our previous study demonstrated that Calpain-7 (CAPN7) is abnormally overexpressed in EMs. Whether CAPN7 affects the regulation of decidualization and by what mechanism CAPN7 regulates decidualization remains to be determined.

\section{Methods}

Immunohistochemistry (IHC) was used to assess the CAPN7 expression in human endometria. Quantitative real-time PCR (qRT-PCR), western blotting, ELIFA and ELISA were applied to explore PRL and IGFBP-1 expressions in decidualized human endometrial stromal cells (HESC). Immunofluorescence analysis and the nuclear and cytoplasmic protein extract assay were employed to test CAPN7's affection on Fox01's location in HESC. Western blotting was used to explore the regulatory mechanism of CAPN7 to AKT1/FoxO1 signalling pathway.

\section{Results}

In this study, we found CAPN7 expression decreased during human endometrial stromal cell (HESC) decidualization in vitro. CAPN7 negatively regulated decidualization in vitro and in vivo. We also identified one conserved potential PEST sequence in the AKT1 protein and found that CAPN7 was able to hydrolyse AKT1 and enhance AKT1's phosphorylation. Correspondingly, CAPN7 notably promoted the phosphorylation of Forkhead Box 01 (Fox01), the downstream of AKT1 protein, at Ser319, leading to increased Fox01 exclusion from nuclei and attenuated FoxO1 transcriptional activity in decidualized HESC. In addition, we detected endometrium CAPN7, p-AKT1 and p-FoxO1 expressions were increased in EMs.

\section{Conclusions}

These data demonstrate that CAPN7 negatively regulates HESC decidualization in EMs probably by promoting Fox01's phosphorylation and Fox01 nuclear exclusion via hydrolyzing AKT1. The dysregulation of CAPN7 may be a novel cause of EMs.

\section{Background}

Endometriosis (EMs) is an oestrogen-dependent disease in which the growth of endometrial tissue (glands and stroma) occurs outside the uterine lining and musculature. Most EMs occur in the pelvic cavity, and their occurrence and development are closely related to infertility in female patients. Studies 
show that $35 \%$ of infertile women have EMs and that $30 \sim 50 \%$ of EMs patients present infertility[1]. The exact mechanism of infertility caused by EMs is still unclear. According to previous reports, damage to endometrial receptivity caused by impaired decidualization may be one of the mechanisms underlying infertility in EMs[2]. Embryo implantation enables endometria to differentiate into deciduas to maintain embryo development and early gestation, which is referred to as decidualization. Some typical morphological and functional changes appear in endometrial stromal cells during decidualization, including a gradually increasing cell size, rounder nuclei and the expression of new proteins such as prolactin (PRL) and insulin-like growth factor binding protein-1 (IGFBP-1)[3, 4].

The transcription factor Fox 01 has been demonstrated to play a vital role in regulating decidualization. Fox01 is of particular importance as a critical regulator of progesterone-dependent differentiation. Decidual protein induced by progesterone (DEPP) is regulated by FoxO1 and FoxO3a at the transcriptional level in human vascular endothelial cells[5]. Fox01 displays a persistent nuclear presence during decidualization both in vivo and in vitro[6]. Fox01 is also essential for the progesterone receptor (PR) binding of IRF4, a novel transcriptional regulator of endometrial stromal decidualization[7]. Moreover, in EMs, the overactivation of the PI3K/Akt pathway reduces the expression of the deciduaspecific gene IGFBP1, probably by decreasing the nuclear Fox01 level[8].

Our previous study demonstrated that CAPN7 was abnormally overexpressed in the endometria of women with EMs[9], suggesting that CAPN7 probably plays a role in regulating decidualization. CAPN7 is a member of the calpain family of Ca2+-dependent cysteine proteases. By hydrolysing substrates, calpains exert regulatory effects in many biological and pathological processes, such as cell proliferation, apoptosis, cell migration and differentiation[10]. Proteins with a polypeptide sequence enriched in proline $(P)$, glutamate $(E)$, serine $(S)$, and threonine $(T)$ (PEST motif) may be targets for degradation by calpains $[11,12]$.

In the present study, we identified one conserved potential PEST sequence in the AKT1 protein and found that CAPN7 negatively regulated HESC decidualization probably by promoting Fox01 nuclear exclusion via hydrolyzing AKT1 and increasing the phosphorylation of AKT1.

\section{Methods}

\section{Patients and sample collection}

Normal endometrial samples were collected from healthy fertile women aged 25-35 years who had no evidence of endometrial abnormalities, endometriosis or adenomyosis. All samples were collected with the informed consent of the patients, and approval from the ethics committee was obtained for this study. During the mid-secretory phase of menstruation, 8-9 days after ovulation, endometrial tissue samples were collected. Eutopic endometrial tissue samples were collected from age-matched patients with laparoscopically diagnosed with EMs. Endometrial biopsy was performed under an approved Human Investigations Committee protocol. All patients enrolled in the study exhibited regular 
menstruation and had no history of hormone treatment before surgery. All patients signed an informed consent form before the operation, and this study was approved by the Drum Tower Hospital Research and Ethics Committee.

\section{Isolation of human endometrial stromal cells (HESCs) and artificial induction of decidualization in vitro}

HESCs were isolated from normal endometrial tissue obtained by endometrial biopsy from normally cycling women who had not received hormone treatment for at least three months. HESCs were isolated as previously described[13]. HESC decidualization in vitro was induced as previously described[14]. For particular treatments, cells were pre-treated with Ad-GFP-CAPN7,Ad-Myc-FOX01, siCAPN7, or si-Ctrl for 2 days and then treated with 8-Br-cAMP and MPA for 3 days.

\section{Immunohistochemistry}

Human uterine endometrial sections were treated as previously described[14] except that the sections were incubated with antibodies against CAPN7 (1:50 dilution, Novus Biologicals, CO, USA, NBP2-

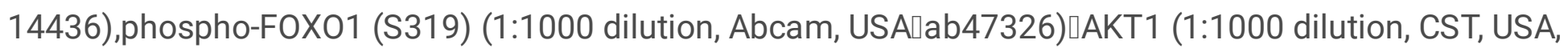
2938S), phospho-Akt (S473) (1:1000 dilution, CST, USA, 4060S) overnight at $4{ }^{\circ} \mathrm{C}$. Control sections were processed concurrently using PBS or rabbit IgG and similarly pretreated. The representative objective protein relative expression level was determined according to the mean and integrated optical density (IOD) of the digital image ( $\times 400)$ according to the software's instructions. In this study, we only calcite the staining score of the endometrium stromal cells.

\section{Western blotting}

HESCs were lysed using whole-cell lysis buffer, and HESC proteins were treated as specified by the instructions of the nuclear and cytoplasmic protein extraction kit (Thermo). Protein concentrations were quantified using the Bradford assay (Bio-Rad Laboratories, Hercules, USA). The proteins $(30 \mu \mathrm{g})$ were separated using 10\% SDS-PAGE and transferred to polyvinylidene fluoride (PVDF) membranes (Millipore, Billerica, USA). The membranes were then blocked for $1 \mathrm{~h}$ in $5 \%$ nonfat milk. Subsequently, the membranes were exposed to primary antibodies against CAPN7 (1:3000 dilution, Novus Biologicals, CO, USA, NBP2-14436), Flag-HRP (1:5000 dilution, Sigma, St. Louis, USA, 7425), GFP (1:1,000; beyotime, Shanghai, China, AG281), FOX01A (1:2000 dilution, Abcam, USA, ab39670), phospho-FOX01 (S319) (1:1000 dilution, Abcam, USA, ab47326), FOX01 (T24) (1:1000 dilution, Abcam, USA, ab58517), phosphoFOXO1 (S256) (1:1000 dilution, Abcam, USA, ab131339), AKT1 (1:1000 dilution, CST, USA, 2938S), phospho-Akt (S473) (1:1000 dilution, CST, USA, 4060S), and HSP90B (1:5000 dilution, Bioworld Technology, St. Louis, USA, BS1181); GAPDH (1:10,000; Bioworld Technology St. Louis, USA, AP0063) or $\beta$-actin (1:5000 dilution, Bioworld Technology, St. Louis, USA, AP0060) was also quantified as an internal 
control. An anti-rabbit secondary antibody, an anti-mouse secondary antibody and an enhanced chemiluminescence kit (Millipore, Billerica, USA) were used for final immunodetection.

\section{RNA isolation and real-time quantitative PCR}

Total RNA was isolated from cells using TRIzol (Life Technologies, New York, USA) according to the manufacturer's instructions. RNA $(1 \mu \mathrm{g})$ was reverse transcribed to obtain CDNA and diluted 1:4 before use. The total reaction volume of $20 \mu \mathrm{L}$ contained $2 \mu \mathrm{L}$ of RNA and contained $2 \mu \mathrm{L}$ of RNA and ChamQ Universal SYBR qPCR Master Mix (Vazyme, Nanjing, China, Q711-02). Real-time PCR analysis was performed with SYBR Green dye and measured on an Analytik Jena instrument to quantify the mRNA levels. The housekeeping gene 18S rRNA was used as an internal control. The specific primers sequences are listed in Table S1.

\section{siRNA knockdown assay}

A pair of small interfering RNA oligonucleotides specific for human CAPN7 (sense strand: 5'CAUUAGUGGUUUCUCAAUAdTdT-3' and 3'-dTdT GUAAUCACCAAAGAGUUAU-5') and a pair of control siRNA oligonucleotides were synthesized by RIBOBIO (Guangzhou, China). At 60-70\% confluence, HESCs were transfected with siRNAs by using SuperFectTMII (Pufei Biotech, Shanghai, China) transfection reagent at a final concentration of $50 \mathrm{nM}$ according to the manufacturer's recommendations.

\section{Prolactin and IGFBP-1 assays}

The HESC supernatant was collected in 6-well plates on the third, sixth, and ninth days after treatment with 8-Br-cAMP and MPA. PRL and IGFBP-1 levels in supernatant were measured using Elecsys Prolactin II (Roche, Switzerland) and Human IGFBP1 ELISA kits (BOSTER, Wuhan, China). The limit of detection of the Vidas Prolactin kit was $0.5 \mathrm{ng} / \mathrm{ml}$, and the detection range of the human IGFBP1 ELISA kit was 31.2$2000 \mathrm{pg} / \mathrm{ml}$. DMEM/F12 supplemented with charcoal/dextran-treated FBS did not contain measurable prolactin or IGFBP-1.

\section{Transient transfection and luciferase reporter assays}

The wild-type IGFBP-1 promoter sequence (-1050 to -113) was amplified by PCR from human endometrial stromal cell genomic DNA with the primers 5'- TATATTATTATCTCGAGACAGGAGGCCCAGGCCTGGAG-3' and 5'- CTGACTGATTAAAGCTTGTTTTGCTAGTGCACCCAAGG-3'. The PCR product was cloned into the pGL3-basic luciferase reporter plasmid (Promega, Madison, WI, USA). The dPRL reporter plasmid was described elsewhere[15]. At 60\% confluence, HESCs in 12-well plates were infected with Ad-CTL or AdFlag-CAPN7 and/or Ad-Flag-FoxO1 for $24 \mathrm{~h}$, and the cells were then transfected with the dPRL reporter plasmid (450 ng/well), IGFBP-1 reporter plasmid (200 ng/well) or IRS (insulin-responsive sequence) 
luciferase reporter plasmid (200 ng/well) (Addgene, MA, USA), together with the Renilla luciferase reporter plasmid pRL-RSV (10 ng) (Promega, Madison, USA), using the Lip2000 transfection reagent (Life Technologies, New York, USA). Six hours after transfection, HESCs were stimulated with 8-Br-cAMP and MPA. After three days, the cell lysates were assayed for luciferase activity. Firefly luciferase activity was normalized for the transfection efficiency according to corresponding Renilla luciferase activity.

\section{Construction of plasmid and adenovirus}

The pCMV-Flag-CAPN7, pEGFP-C1-CAPN7, pCMV-Flag-AKT1 and pEGFP-N1-AKT1 plasmids were obtained by cloning the CAPN7 or AKT1 cDNA obtained by PCR amplification from total human CDNA, which was subcloned into the BgllI- BamHII sites of a pFIAG-CMV, pEGFP-C1/N1 backbone plasmid. The FoxO1 cDNA PCR fragment was digested with the Xhol and Xbal restriction enzymes and cloned in a pCS2-myc plasmid digested in a similar manner. The specific primers are listed in table S2. All of the vectors were examined and verified by restriction analysis and sequencing. The adenoviruses were packaged and amplified as previously described[16].

\section{Coimmunoprecipitation}

For the coimmunoprecipitation (co-IP) of CAPN7 and Fox01 or CAPN7 and AKT1, HEK293T cells were cotransfected with the indicated expression plasmids, and cell lysates were prepared 2 days post transfection. Equal amounts of total proteins $(600 \mu \mathrm{g})$ were precleared by incubation with protein $A / G$ plus-agarose beads (Abmart, Shanghai, China, A10001) and gently shaken at $4{ }^{\circ} \mathrm{C}$ for $2 \mathrm{~h}$. The supernatant was incubated with Flag antibody-conjugated beads (Sigma, St. Louis, USA, M8823) at $4{ }^{\circ} \mathrm{C}$ overnight with gentle shaking, followed by four washes in $1 \mathrm{ml}$ of lysis buffer. The immunocomplex was separated by SDS-PAGE, and the proteins were detected with the indicated antibodies. The Co-IP of GFPCAPN7 and endogenous AKT1 and Fox01 was performed using the total proteins (1 mg) from decidualized HESCs infected with Ad-GFP-CAPN7. The proteins were then treated as described above

\section{Immunofluorescence staining}

HESCs plated on $18 \mathrm{~mm}$ micro-cover glasses (Matsunami, Osaka, Japan) were infected with Ad-GFPCAPN7 and then treated with or without 8-Br-CAMP plus MPA for $60 \mathrm{~h}$. Subsequently, HESCs were treated as previously described[9] except that the cells were incubated with an anti-Fox01 antibody (Cell Signalling Technology, Beverly, Massachusetts, USA, 2880S) to label endogenous Fox01 protein at $4{ }^{\circ} \mathrm{C}$ overnight, and Alexa Fluor 594-conjugated goat anti-rabbit IgG (Life Technologies, New York, USA, A11012) was applied on the second day. The results were visualized using an FV10i-LIV/FV10i-DOC confocal laser scanning biological microscope (Olympus Corporation, Shinjuku, Tokyo, Japan). 


\section{Artificial induction of decidualization}

All of the experimental procedures were performed according to the guidelines of the Experimental Animal Management Committee (Jiangsu Province, China) and were approved by the Ethics Review Board for Animal Studies of Drum Tower Hospital, Nanjing University Medical School. Four- to six-weekold female ICR mice were purchased from the Nanjing Biomedical Research Institute of Nanjing University. The mice were housed in a room maintained under IVC constant environmental conditions, with a temperature of $22-24^{\circ} \mathrm{C}$ and a 12-hr light/dark cycle. Mice at 6-8 wk of age were ovariectomized under isoflurane anaesthesia and $20 \mu \mathrm{l}(2 \times 108 \mathrm{TU} /$ side $)$ of Ad-GFP or Ad-GFP-CAPN7 were injected into the uterine lumen. After $1 \mathrm{wk}$, Ad-GFP-CAPN7 was injected into the tail veins of the mice, and Ad-lacZ was injected as a control. After an additional $1 \mathrm{wk}$ to allow endogenous ovarian hormones to disappear completely, the mice were subjected s.c. injections of E2 (100 ng) daily for $3 \mathrm{~d}$. After $2 \mathrm{~d}$ of rest, the mice were treated with $1 \mathrm{mg}$ of P4 s.c. and $10 \mathrm{ng}$ of E2 s.c. daily for $3 \mathrm{~d}$. A second surgery was performed $6 \mathrm{~h}$ after the last hormone injection to expose one uterine horn. Decidualization was induced in this horn by the injection of $20 \mu \mathrm{L}$ of sesame oil into (sigma)the lumen. The other uterine horn was left untreated as a control. Daily treatments (1 mg of P4 s.c. and $10 \mathrm{ng}$ of E2 s.c.) were continued for $4 \mathrm{~d}$. The mice were killed $6 \mathrm{~h}$ after the last oil injection, and the wet weights of the traumatized and control uterine horns of each mouse were recorded. Uterine tissue was collected from both horns and fixed in $10 \%$ neutral buffered formalin[17].

\section{Statistical analysis}

Unless stated otherwise, the presented numerical data are the mean \pm SD from at least three experiments. Statistical analyses were performed using Prism version 6 software. Statistical differences in the mean expression values of the two treatment groups were compared using a two-tailed Student's $t$-test. Oneway ANOVA was performed for comparisons among more than two groups.

\section{Results}

\section{CAPN7 decreases during HESC decidualization}

Immunohistochemistry demonstrated that CAPN7 protein expression was obviously decreased in human secretory-phase endometria compared with that in proliferative-phase endometria (Fig. 1a). To determine CAPN7 expression during HESC decidualization, we used 8-bromoadenosine-cAMP (8-Br-CAMP) and medroxyprogesterone acetate (MPA) to induce HESC decidualization in vitro and then investigated the dynamic changes in CAPN7 expression in HESCs. As shown in Fig. 1b, 8-Br-cAMP and MPA attenuated CAPN7 protein expression in HESCs in a time-dependent manner.

\section{CAPN7 negatively regulates decidualization in vitro and in vivo}


Next, we studied the role of CAPN7 in HESC decidualization regulation by determining the expression of PRL and IGFBP-1, two decidualization marker genes. As shown in Fig. 2b and 2c, in decidualized HESCs, CAPN7 overexpression inhibited the transcriptional activation of the PRL and IGFBP-1 promoters by approximately $85 \%$ and $75 \%$, respectively. PRL and IGFBP- 1 mRNA expression induced by 8-Br-cAMP and MPA was downregulated by approximately $20 \%$ and $80 \%$ by CAPN7 overexpression, respectively. (Fig. 2d, 2e). Fig. $2 \mathrm{f}, 2 \mathrm{~g}$ shows that CAPN7 overexpression notably suppressed PRL and IGFBP-1 secretion in a time-dependent manner $(P<0.01)$.

To further confirm the functional significance of the role of CAPN7 in the regulation of the two decidualization marker genes, we performed functional deletion studies employing RNA interference. Fig. $2 \mathrm{~h}$ and $2 \mathrm{i}$ show that endogenous CAPN7 expression was markedly suppressed when HESCs were transfected with Si-CAPN7 at the indicated concentration for three days $(P<0.01)$. As expected, CAPN7 knockdown strongly promoted PRL and IGFBP-1 mRNA expression by approximately 4-fold in decidualized HESCs (Fig. 2j, 2k). In addition, CAPN7 knockdown upregulated PRL and IGFBP-1 secretion mediated by 8 -Br-cAMP and MPA in a time-dependent manner $(P<0.01)$ (Fig. $2 \mathrm{l}, 2 \mathrm{~m})$.

We also studied the role of CAPN7 in decidualization in vivo using an artificial decidualization experiment. The hormonal profile of pregnancy is simulated in ovariectomized mice, and decidualization is triggered by the injection of sesame seed oil into the uterine lumen of one of the two uterine horns[17]. As shown in the Fig. 2n, the morphological analysis of Ad-LacZ control mice revealed thicker uterine horns, indicating the presence of pronounced deciduoma after artificial stimulation. In contrast, the uteri of CAPN7-overexpressing mice contained thinner and fewer pseudopregnant sacs, suggesting that the Ad-CAPN7-treated mice showed a significantly diminished response to decidual stimuli.

\section{CAPN7 suppresses the mRNA expression of Fox01 target genes}

It has been well documented that the transcription factor Fox01 plays a vital role in regulating decidualization[5-8]; therefore, we examined whether CAPN7 influenced the transcriptional activity of Fox01. As shown in Figure. 3b, CAPN7 notably attenuated the transcriptional activity of Fox01 in decidualized HESCs. PRL, IGFBP-1, decorin (DCN), and tissue metalloproteinase inhibitor-3 (TIMP-3) are specific genes associated with decidualization downstream of Fox01[5]. CAPN7 obviously decreased the mRNA expression of PRL, IGFBP-1 and DCN induced by FoxO1 in decidualized HESCs $(P<0.01)$, indicating that CAPN7 was able to inhibit the function of Fox01 (Fig. 3c-f).

\section{CAPN7 promotes Fox01 nuclear exclusion in decidualized HESCs}


To further explore the mechanism by which CAPN7 affects Fox01 function, we examined whether CAPN7 interacts with Fox01. Immunoprecipitation assays demonstrated that Flag-CAPN7 associated with MycFox01 in HEK293T cells (Fig. 4a). However, we did not detect an obvious reduction in FoxO1 expression caused by CAPN7 overexpression in HESCs (data not shown).

Interestingly, immunofluorescence analysis demonstrated that CAPN7 affects FoxO1 localization in decidualized HESCs. As shown in Fig. 4b, the characteristic morphological changes associated with decidualization appeared in decidualized HESCs that were not infected with Ad-GFP-CAPN7, and the majority of Fox01 entered nuclei after 8-Br-cAMP and MPA stimulation. However, in Ad-GFP-CAPN7infected HESCs, Fox01 was mainly localized in the cytoplasm, and its entry into nuclei was sharply attenuated in decidualized HESCs, which was further confirmed by the nuclear and cytoplasmic protein extract assay, indicating that CAPN7 overexpression obviously promoted Fox01 nuclear exclusion in decidualized HESCs (Fig. 4c).

\section{CAPN7 hydrolyses AKT1 and enhances the phosphorylation of Fox01 and AKT1 in decidualized HESCs}

Previous studies suggested that calpain members usually hydrolyse their substrates to influence their functions $[13,18]$. However, in our research, Capn7 affected the function of Fox01 without hydrolysing the Fox01 protein. Therefore, we speculated that CAPN7 might indirectly influence FoxO1 function through other mechanisms. The function of FoxO1 is controlled by a wide range of posttranslational modifications, such as phosphorylation, and the kinase AKT1 is one of the most important upstream proteins of Fox01[19]. Therefore, the interaction between CAPN7 and AKT1 was detected (Fig. 5a). We identified one conserved potential PEST sequence in AKT1 by using the ePESTfind program (emboss.bioinformatics.nl). The PEST sequence was located in the C-terminus outside of the kinase domain in AKT1 (Fig. 5b). Fig. 5c demonstrates that with an increasing CAPN7 concentration, the expression of AKT1-GFP decreased and the amount of the cleavage product increased, suggesting that CAPN7 was able to hydrolyse AKT1 in a concentration-dependent manner. Meanwhile, as the CAPN7 concentration increased, the expression of AKT1 $\triangle P E S T$ GFP didn't decrease and there wasn't any cleavage product (Fig. 5d). Endogenous IP demonstrated that Capn7 overexpression was able to enhance Fox01's binding to AKT1 in decidualized HESC (Fig. 5e). In addition, western blotting was employed to determine the activity of the AKT1/Fox01 signalling pathway. As shown in Fig. 5f, CAPN7 overexpression obviously promoted Fox01 phosphorylation at Ser319, Ser256 and Thr24 in decidualized HESC, which could explain the increase in Fox01 nuclear exclusion. Among these three phosphorylation sites, the enhancement of Fox01's phosphorylation at Ser319 was the most significant and the change was still quite obvious even in HESC without the treatment of $8 \mathrm{Br}$ and MPA. CAPN7 overexpression also significantly increased AKT1 phosphorylation at Ser473. 


\section{Endometrium CAPN7, p-AKT1 and p-Fox01 expression is increased in EMs}

Immunohistochemistry analysis showed that CAPN7, AKT1, p-AKT1 and p-Fox01(Ser319) were expressed in both stromal and epithelial tissues. Significantly higher endometrium expression levels of CAPN7, p-AKT1 and p-Fox01(Ser319) were detected in the in the EMs group than in the normal group, especially in stromal cells $(P<0.01)$. The expression of AKT1 was not significantly different between the EMs group and the control group (Fig. 6a). The above results were consistent with the western blot analysis and suggested that in EMs, overexpressed CAPN7 probably promoted the phosphorylation of AKT1, facilitating FoxO1 phosphorylation and nuclear exclusion, ultimately inhibiting decidualization.

\section{Discussion}

This study demonstrated that CAPN7 inhibited decidualization by promoting Fox01 nuclear exclusion by hydrolysing and activating AKT1 to increase the phosphorylation of FoxO1.

As a family of $\mathrm{Ca}^{2+}$-dependent cysteine proteases, calpains hydrolyse substrates when activated by $\mathrm{Ca}^{2+}$ at proper concentrations to take part in the regulation of biological movements in cells[10, 20, 21]. Some previous studies have demonstrated that members of the calpain family participate in regulating the endometrial decidualization process. For instance, $\mu$-calpain expression is much higher in the decidua of women with recurrent miscarriage than in the decidua of healthy women[22]. Calpain-2 activity increases at the time of implantation in rat uterine luminal epithelial cells, and the administration of a calpain inhibitor significantly reduces the number of implantation sites, suggesting that Calpain-2 plays an important role in modulating endometrial receptivity for implantation[23]. Our previous study demonstrated that CAPN7 impaired embryo implantation by downregulating $\beta 3$-integrin expression by degrading HOXA10 in endometrial epithelial cells in EMs[14]. Similarly, this study showed that in EMs, CAPN7 inhibited decidualization by hydrolysing AKT1 in endometrial stromal cells. Moreover, we found that CAPN7 overexpression increased AKT1 phosphorylation at Ser473, and in the EMs group, the expression of both CAPN7 and p-AKT1 (Ser473) was abnormally increased, which was consistent with the results of previous studies. Cinar revealed higher pAkt (Ser473) immunoreactivity in eutopic and ectopic endometria than in normal endometria[24]. Ruiz demonstrated that CXCL12 promoted the proliferation, migration, and invasion of endometriotic cells by inducing AKT serine 473 phosphorylation[25]. Madane also found that the expression of pAkt (Ser473) was higher in in an EMs group than in the control group[26]. In the present study, we also identified one conserved PEST sequence as a potential target for degradation by calpains, which is located in the C-terminus and outside the kinase domain in AKT1. We hypothesized that the cleavage of AKT1 by CAPN7 exposed the phosphorylation site of AKT1, facilitating AKT1 phosphorylation and activation.

Fox01, the downstream protein of AKT1, has been identified as a gene that is upregulated early in the decidualization process and is able to increase both PRL and IGFBP-1 promoter activities in HESCs[5, 27] and human decidual fibroblasts[28]. Fox01 is also reported to be closely associated with progesterone 
signalling. EMs patients usually show obvious progesterone resistance, and decreases in several transcription factors containing FoxO1 play an important role in this resistance[29]. FoxO1 is also involved in the endometrial receptivity regulation in adenomyosis[30]. This study suggested that CAPN7 could affect Fox01 function without hydrolysing the Fox01 protein. Moreover, immunofluorescence analysis showed that CAPN7 affects the cellular localization of FoxO1 in decidualized HESCs. Therefore, we speculated that CAPN7 might indirectly influence FoxO1 function through other mechanisms. We further examined the phosphorylation status of Fox01 and found that CAPN7 overexpression markedly promoted Fox01 phosphorylation at Ser319, Ser256 and Thr24, which could explain the increased nuclear exclusion and depressed transcriptional function of Fox01. The function of FoxO1 is controlled by a wide range of posttranslational modifications, including phosphorylation, acetylation and ubiquitination, in which phosphorylases such as AKT, serum- and glucocorticoid-inducible kinase (SGK), acetylases such as p300/CBP associated factor (PCAF) and some cofactors play critical roles[31-33]. Fox01 phosphorylation depends on the activity of its upstream phosphorylases, such as AKT1, casein kinase 1 and p21/Cdc42/Rac1-activated kinase 1 (PAK1). According to previous studies, AKT1 is activated after phosphorylation and can then phosphorylate Fox01 at Ser319, Ser256 and Thr24, while PAK1 phosphorylates FoxO1 at Ser256[34]. Fox01 phosphorylation induces Fox01 binding to 14-3-3 proteins and then translocates the resulting complex to the cytosol, where the bound 14-3-3 proteins prevent the reentry of FoxO1 into nuclei, likely by interfering with the function of its nuclear localization signal (NLS) and ultimately inhibiting the transcriptional activity of Fox01[19, 35]. In this study, although we clarified that CAPN7 could affect Fox01 phosphorylation by hydrolysing and activating AKT1, the effect of PAK1, another possible upstream kinase of Fox01, was not studied. The above limitation suggests that further in-depth analysis is still needed in the future.

In conclusion, our study revealed that CAPN7 acts as a novel repressor in HESC decidualization and preliminarily showed that CAPN7 inhibits HESC decidualization, likely by promoting FoxO1 phosphorylation and nuclear exclusion by increasing AKT1 phosphorylation, resulting in impaired endometrial receptivity in EMs. Furthermore, CAPN7 might become a potential target for the treatment of decidualization-associated diseases such as recurrent miscarriage and EMs.

\section{Abbreviations}

CAPN7: Calpain7; Fox01: Forkhead Box 01; AKT1: AKT serine/threonine kinase 1; EMs: endometriosis; IHC: Immunohistochemistry; HESC: human endometrial stromal cell; PRL: prolactin; IGFBP-1: insulin-like growth factor binding protein-1; IOD: integrated optical density; 8-Br-cAMP: 8-bromoadenosine-cAMP; MPA: medroxyprogesterone acetate; PCAF: p300/CBP associated factor; qRT-PCR: Quantitative real-time PCR; PR: progesterone receptor; PVDF: polyvinylidene fluoride; IRS: insulin-responsive sequence; co-IP: coimmunoprecipitation; DCN: decorin; TIMP-3: tissue metalloproteinase inhibitor-3; SGK: serum- and glucocorticoid-inducible kinase; PAK1: p21/Cdc42/Rac1-activated kinase 1; NLS: nuclear localization signal. 


\section{Declarations}

\section{Ethical Approval and Consent to participate}

The use of human tissues was approved by the institutional review board of the Drum Tower Hospital of Nanjing University on the 5th of December 2013 (2013-081-01). Patient consents were received before biopsy, and the study was performed in accordance with the Declaration of Helsinki.

\section{Consent for publication}

All co-authors have seen and approved the fnal version of the paper and have agreed to its submission for publication. All patients signed informed written consent forms.

\section{Competing interests}

We declare no potential conflicts of interest concerning the research, authorship, and/or publication of this article.

\section{Funding}

This work was supported by the National Key Research and Development Program of China (2018YFC1004400, NO. 82030040), the National Natural Science Foundation of China (No. 81070492, No. 81871165 , No. 31872846 , NO. 81971387, NO. 82071646, NO.81801530).

\section{Authors' contributions}

Haixiang Sun and Guijun Yan supervised the project and designed, edited, and led the experiments of this study. Nannan Kang, Huizhi Shan, Jidong Zhou, Chenyang Huang, Hui Zhang, Mei Zhang and Xin Zhen conducted the experiments and data analysis. Nannan Kang and Huizhi Shan organized the public data and prepared all the figures and tables and drafted the manuscript. Junxia Wang and Jie Mei revised the manuscript. All the authors reviewed and approved the final manuscript.

\section{Acknowledgements}

The National Natural Science Foundation of China is gratefully acknowledged.

\section{References}

1. de Ziegler D, Borghese B, Chapron C: Endometriosis and infertility: pathophysiology and management. Lancet 2010, 376:730-738.

2. Su RW, Strug MR, Joshi NR, Jeong JW, Miele L, Lessey BA, Young SL, Fazleabas AT: Decreased Notch pathway signaling in the endometrium of women with endometriosis impairs decidualization. J Clin Endocrinol Metab 2015, 100:E433-442. 
3. Ganeff C, Chatel G, Munaut C, Frankenne F, Foidart JM, Winkler R: The IGF system in in-vitro human decidualization. Mol Hum Reprod 2009, 15:27-38.

4. Ramathal CY, Bagchi IC, Taylor RN, Bagchi MK: Endometrial decidualization: of mice and men. Semin Reprod Med 2010, 28:17-26.

5. Buzzio OL, Lu Z, Miller CD, Unterman TG, Kim JJ: FOX01A differentially regulates genes of decidualization. Endocrinology 2006, 147:3870-3876.

6. Ujvari D, Jakson I, Babayeva S, Salamon D, Rethi B, Gidlöf S, Hirschberg AL: Dysregulation of In Vitro Decidualization of Human Endometrial Stromal Cells by Insulin via Transcriptional Inhibition of Forkhead Box Protein 01. PLoS One 2017, 12:e0171004.

7. Vasquez YM, Mazur EC, Li X, Kommagani R, Jiang L, Chen R, Lanz RB, Kovanci E, Gibbons WE, DeMayo FJ: FOXO1 is required for binding of PR on IRF4, novel transcriptional regulator of endometrial stromal decidualization. Mol Endocrinol 2015, 29:421-433.

8. Yin X, Pavone ME, Lu Z, Wei J, Kim JJ: Increased activation of the PI3K/AKT pathway compromises decidualization of stromal cells from endometriosis. J Clin Endocrinol Metab 2012, 97:E35-43.

9. Liu H, Jiang Y, Jin X, Zhu L, Shen X, Zhang Q, Wang B, Wang J, Hu Y, Yan G, Sun H: CAPN 7 promotes the migration and invasion of human endometrial stromal cell by regulating matrix metalloproteinase 2 activity. Reprod Biol Endocrinol 2013, 11:64.

10. Goll DE, Thompson VF, Li H, Wei W, Cong J: The calpain system. Physiol Rev 2003, 83:731-801.

11. Rechsteiner M, Rogers SW: PEST sequences and regulation by proteolysis. Trends Biochem Sci 1996, 21:267-271.

12. Martinez LO, Agerholm-Larsen $B$, Wang $N$, Chen W, Tall AR: Phosphorylation of a pest sequence in ABCA1 promotes calpain degradation and is reversed by ApoA-I. J Biol Chem 2003, 278:3736837374.

13. Zyskind JW, Wang Y, Cho G, Ting JH, Kolson DL, Lynch DR, Jordan-Sciutto KL: E2F1 in neurons is cleaved by calpain in an NMDA receptor-dependent manner in a model of HIV-induced neurotoxicity. J Neurochem 2015, 132:742-755.

14. Yan Q, Huang C, Jiang Y, Shan H, Jiang R, Wang J, Liu J, Ding L, Yan G, Sun H: Calpain7 impairs embryo implantation by downregulating $\beta 3$-integrin expression via degradation of HOXA10. Cell Death Dis 2018, 9:291.

15. Jiang Y, Hu Y, Zhao J, Zhen X, Yan G, Sun H: The orphan nuclear receptor Nur77 regulates decidual prolactin expression in human endometrial stromal cells. Biochem Biophys Res Commun 2011, 404:628-633.

16. Zhou Q, Yan G, Ding L, Liu J, Yu X, Kong S, Zhang M, Wang Z, Liu Y, Jiang Y, et al: EHD1 impairs decidualization by regulating the Wnt4/ $\beta$-catenin signaling pathway in recurrent implantation failure. EBioMedicine 2019, 50:343-354.

17. Zhang H, Zhu X, Chen J, Jiang Y, Zhang Q, Kong C, Xing J, Ding L, Diao Z, Zhen X, et al: Krüppel-like factor 12 is a novel negative regulator of forkhead box 01 expression: a potential role in impaired decidualization. Reprod Biol Endocrinol 2015, 13:80. 
18. Wu SM, Lin WY, Shen CC, Pan HC, Keh-Bin W, Chen YC, Jan YJ, Lai DW, Tang SC, Tien HR, et al: Melatonin set out to ER stress signaling thwarts epithelial mesenchymal transition and peritoneal dissemination via calpain-mediated C/EBPß and NFKB cleavage. J Pineal Res 2016, 60:142-154.

19. Saline M, Badertscher L, Wolter M, Lau R, Gunnarsson A, Jacso T, Norris T, Ottmann C, Snijder A: AMPK and AKT protein kinases hierarchically phosphorylate the N-terminus of the F0X01 transcription factor, modulating interactions with 14-3-3 proteins. J Biol Chem 2019, 294:1310613116.

20. Siklos M, BenAissa M, Thatcher GR: Cysteine proteases as therapeutic targets: does selectivity matter? A systematic review of calpain and cathepsin inhibitors. Acta Pharm Sin B 2015, 5:506-519.

21. Yang J, Xiang F, Cai PC, Lu YZ, Xu XX, Yu F, Li FZ, Greer PA, Shi HZ, Zhou Q, et al: Activation of calpain by renin-angiotensin system in pleural mesothelial cells mediates tuberculous pleural fibrosis. Am J Physiol Lung Cell Mol Physiol 2016, 311:L145-153.

22. Kumagai K, Ozaki Y, Nakanishi T, Inomata M, Furuno T, Nakanishi M, Ogasawara MS: Role of mucalpain in human decidua for recurrent miscarriage. Am J Reprod Immunol 2008, 59:339-346.

23. Kaneko Y, Murphy CR, Day ML: Calpain 2 activity increases at the time of implantation in rat uterine luminal epithelial cells and administration of calpain inhibitor significantly reduces implantation sites. Histochem Cell Biol 2014, 141:423-430.

24. Cinar O, Seval Y, Uz YH, Cakmak H, Ulukus M, Kayisli UA, Arici A: Differential regulation of Akt phosphorylation in endometriosis. Reprod Biomed Online 2009, 19:864-871.

25. Ruiz A, Ruiz L, Colón-Caraballo M, Torres-Collazo BJ, Monteiro JB, Bayona M, Fazleabas AT, Flores I: Pharmacological blockage of the CXCR4-CXCL12 axis in endometriosis leads to contrasting effects in proliferation, migration, and invasion. Biol Reprod 2018, 98:4-14.

26. Madanes D, Bilotas MA, Bastón JI, Singla JJ, Meresman GF, Barañao RI, Ricci AG: PI3K/AKT pathway is altered in the endometriosis patient's endometrium and presents differences according to severity stage. Gynecol Endocrinol 2020, 36:436-440.

27. Kim JJ, Taylor HS, Akbas GE, Foucher I, Trembleau A, Jaffe RC, Fazleabas AT, Unterman TG: Regulation of insulin-like growth factor binding protein-1 promoter activity by FKHR and HOXA10 in primate endometrial cells. Biol Reprod 2003, 68:24-30.

28. Christian M, Zhang X, Schneider-Merck T, Unterman TG, Gellersen B, White JO, Brosens JJ: Cyclic AMP-induced forkhead transcription factor, FKHR, cooperates with CCAAT/enhancer-binding protein beta in differentiating human endometrial stromal cells. J Biol Chem 2002, 277:20825-20832.

29. Marquardt RM, Kim TH, Shin JH, Jeong JW: Progesterone and Estrogen Signaling in the Endometrium: What Goes Wrong in Endometriosis? Int J Mol Sci 2019, 20.

30. Tian J, Zhang C, Kang N, Wang J, Kong N, Zhou J, Wu M, Ding L, Sun H, Yan G, Sheng X: Attenuated monoamine oxidase a impairs endometrial receptivity in women with adenomyosis via downregulation of F0X01. Biol Reprod 2021.

31. Matsuzaki H, Daitoku H, Hatta M, Aoyama H, Yoshimochi K, Fukamizu A: Acetylation of Foxo1 alters its DNA-binding ability and sensitivity to phosphorylation. Proc Natl Acad Sci U S A 2005, 102:11278- 
11283.

32. Huang $H$, Regan KM, Wang F, Wang D, Smith DI, van Deursen JM, Tindall DJ: Skp2 inhibits FOXO1 in tumor suppression through ubiquitin-mediated degradation. Proc Natl Acad Sci U S A 2005, 102:1649-1654.

33. Yoshimochi K, Daitoku H, Fukamizu A: PCAF represses transactivation function of FOXO1 in an acetyltransferase-independent manner. J Recept Signal Transduct Res 2010, 30:43-49.

34. Mazumdar A, Kumar R: Estrogen regulation of Pak1 and FKHR pathways in breast cancer cells. FEBS Lett 2003, 535:6-10.

35. Gerst F, Kaiser G, Panse M, Sartorius T, Pujol A, Hennige AM, Machicao F, Lammers R, Bosch F, Häring $\mathrm{HU}$, Ullrich S: Protein kinase C $\delta$ regulates nuclear export of FOXO1 through phosphorylation of the chaperone 14-3-37. Diabetologia 2015, 58:2819-2831.

\section{Figures}




\section{Capn7 decreases during HESC decidualization}

\section{a}

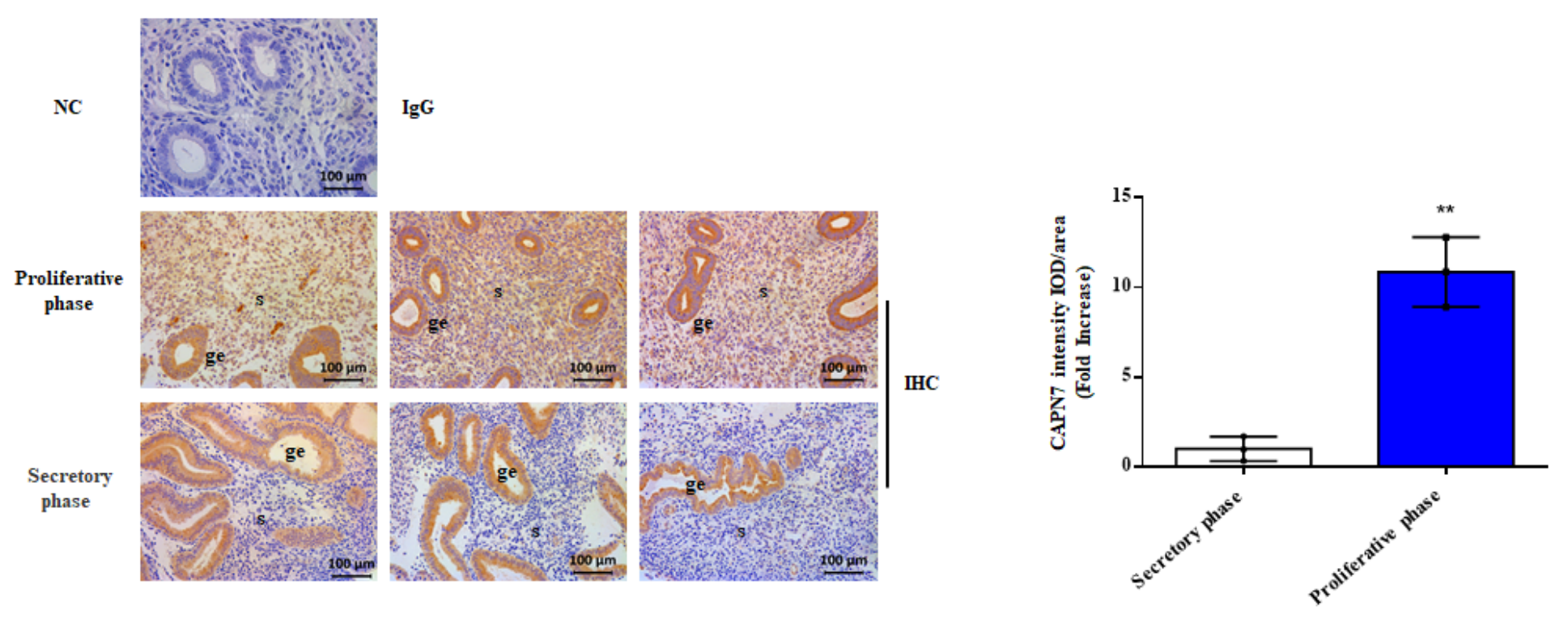

b
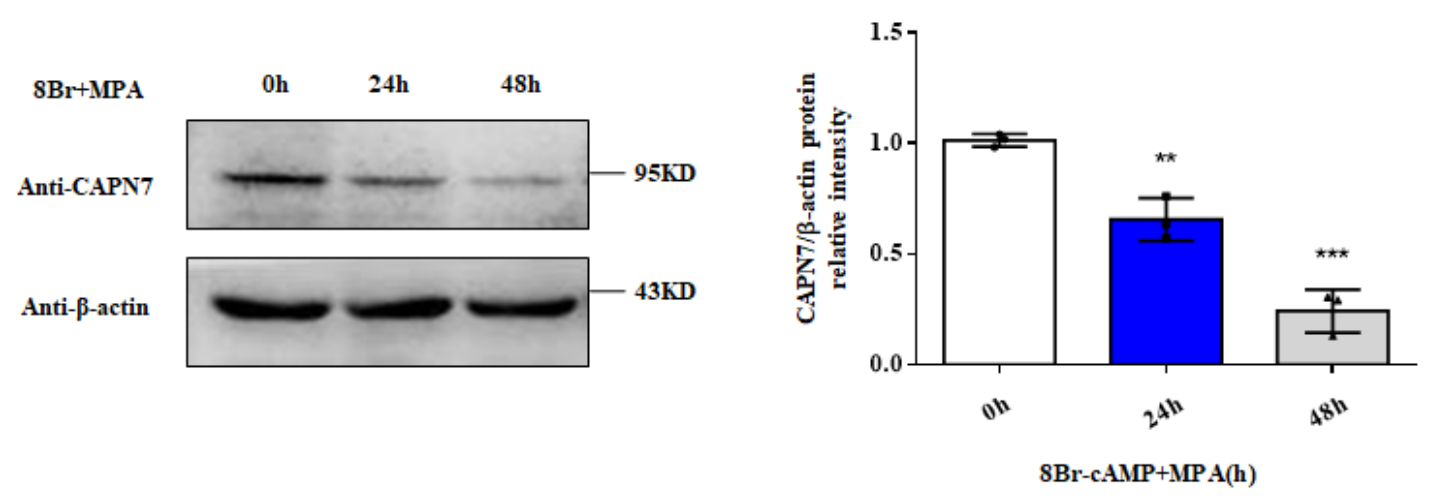

Figure 1

CAPN7 decreases during HESC decidualization. (a) Immunohistochemical detection of CAPN7 in human endometrium during the proliferative phase and the secretory phases of the menstrual cycle using CAPN7 antibody. All images are at $\times 200$ magnification. Scale bars: $100 \mu \mathrm{m}$. ${ }^{\star \star} \mathrm{p}<0.01$ compared with the secretory group (t-test). (b) Total proteins were isolated from HESC treated with 8-Br-cAMP and MPA at the indicated concentrations for different periods. CAPN7 protein expression during HESC decidualization in vitro was assessed by Western Blot. ${ }^{* *} p<0.01$ compared with the Oh group. ${ }^{* \star} p<0.001$ compared with the Oh group (t-test). 
a

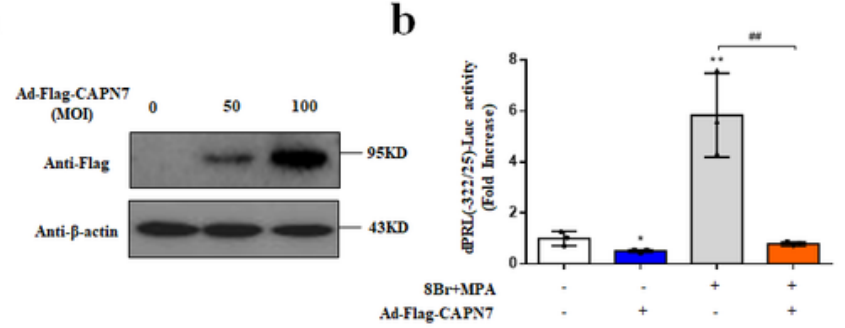

c

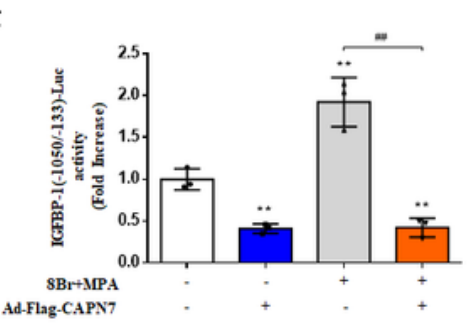

d

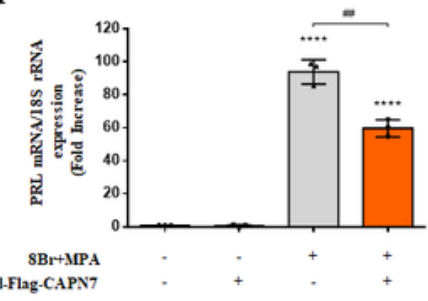

e

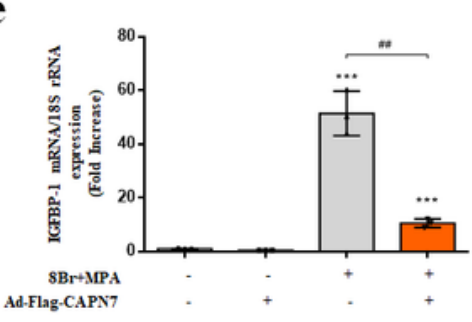

h

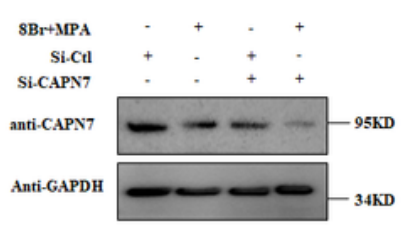

k

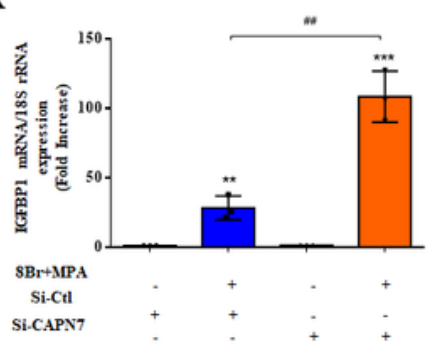

f ${ }^{120}$

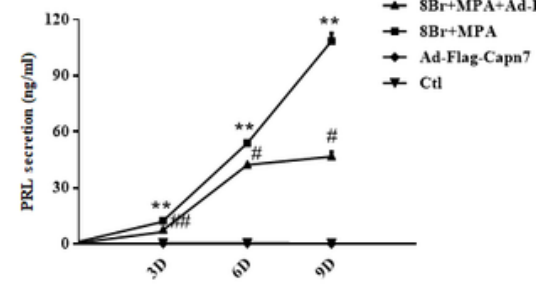

i

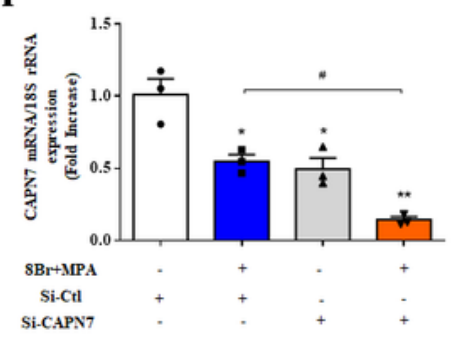

l

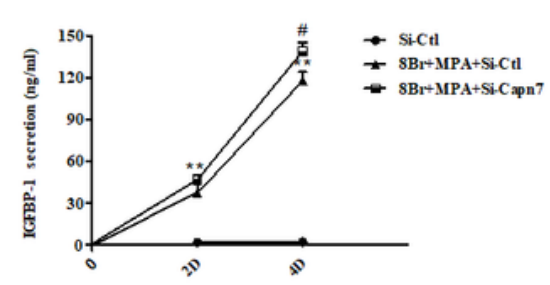

j g
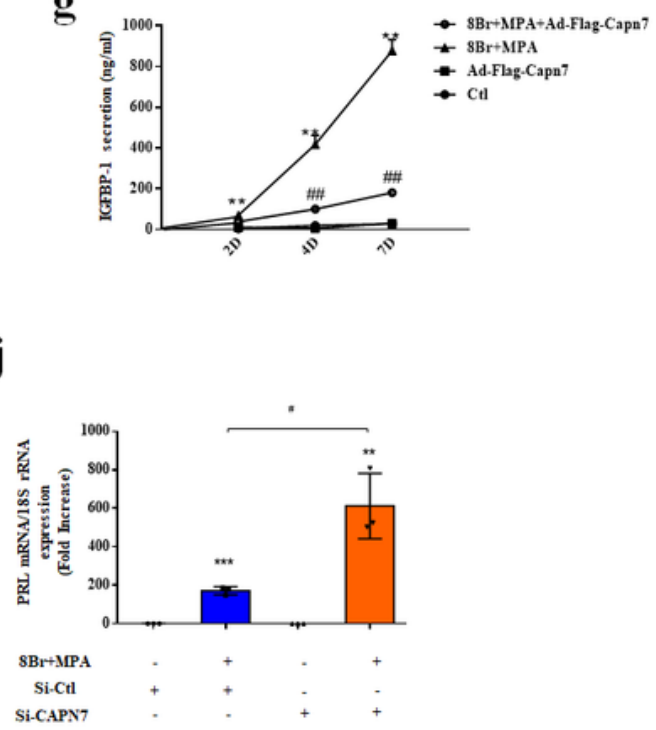

m

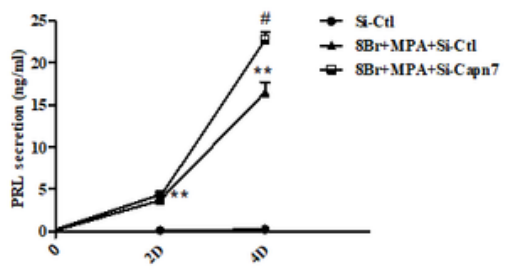

n

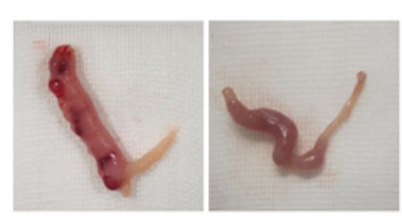

Ad.LacZ

Ad-GFP-CAPN7

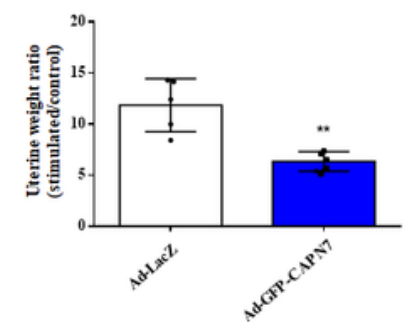

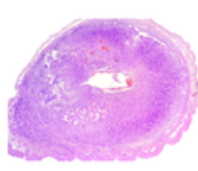

Ad.LacZ

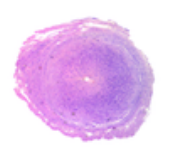

Ad-GFP-CAPN7

Figure 2

CAPN7 negatively regulates decidualization in vitro and in vivo. (a) HESC were infected with Ad-LacZ or Ad-Flag-CAPN7 at the MOI indicated. Flag-CAPN7 protein expression was measured using Western blot. (b) HESC were infected with Ad-LacZ or Ad-Flag-CAPN7 at the MOI indicated for $24 \mathrm{~h}$. Cells were then transfected with the PRL(-332/65)-Luc reporter construct using Lipo2000 transfection reagent, followed by treatment with or without 8-Br-cAMP and MPA. After $48 \mathrm{~h}$, luciferase assays were performed and 
normalized by constitutive Renilla luciferase. $\left(n=3,{ }^{*} p<0.05\right.$, ${ }^{\star *} p<0.01$, \#\# $\left.p<0.01\right)$. (c) HESC were infected with Ad-LacZ or Ad-Flag-CAPN7 at the MOI indicated for $24 \mathrm{~h}$. Cells were then transfected with the IGFBP-1(-1050/-113)-Luc reporter construct using Lipo2000 transfection reagent, followed by treatment with or without 8-Br-cAMP and MPA. After $48 \mathrm{~h}$, luciferase assays were performed and normalized by constitutive Renilla luciferase. $(n=3, * \star p<0.01$, \#\# $p<0.01)$. (d) HESC were infected with Ad-LacZ or Ad-Flag-CAPN7 at the MOI indicated and treated with 8-Br-cAMP plus MPA for two days, after which PRL mRNA levels were measured using real-time PCR. $(n=3, \star \star \star \star ~ p<0.0001$, \#\# $p<0.01)$. (e) HESC were infected with Ad-LacZ or Ad-Flag-CAPN7 at the MOI indicated and treated with 8-Br-cAMP plus MPA for two days, after which IGFBP-1 mRNA levels were measured using real-time PCR. $(n=3, \star \star \star ~ p<$ 0.001 , \#\# $p<0.01$ ). (f) HESC were infected with the indicated adenoviruses for $24 \mathrm{~h}$, followed by treatment with or without 8-Br-cAMP and MPA for an additional three, six, or nine days. PRL secreted into the medium were measured using ELIFA. $(n=3, * \star P<0.01$ compared with Ad-LacZ alone; $\# P<0.05$, \#\# $P<0.01$ ). (g) HESC were infected with the indicated adenoviruses for $24 \mathrm{~h}$, followed by treatment with or without 8-Br-cAMP and MPA for an additional two, four, or seven days. IGFBP-1 secreted into the medium were measured using ELISA. $(n=3, * \star P<0.01$ compared with Ad-LacZ alone; \#\# $P<0.01$ compared with Ad-LacZ treated with 8-Br-cAMP and MPA). (h), (i) Western Blot and real-time PCR show expression of CAPN7 in HESC transfected with Si-Ctl or Si-CAPN7 at indicated concentration. $(n=3, * p<0.05$, ** $p<$ 0.01 , \#\# $p<0.01$ ). (j) HESC were transfected with Si-Ctl or Si-CAPN7 at indicated concentration followed by treatment with or without 8-Br-cAMP and MPA for three days. PRL mRNA levels were measured by realtime PCR. $(n=3, * \star p<0.01, \star \star \star ~ p<0.001, \# p<0.05$,). (k) HESC were transfected with Si-Ctl or Si-CAPN7 at indicated concentration followed by treatment with or without 8-Br-cAMP and MPA for three days.

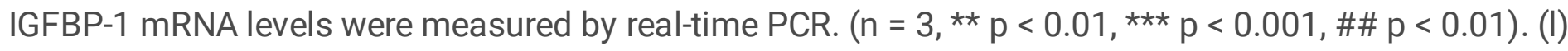
HESC were transfected with Si-Ctl or Si-CAPN7 at indicated concentration, followed by treatment with or without 8-Br-cAMP and MPA for an additional two or four days. IGFBP-1 secreted into the medium were measured using ELISA. $(n=3, * * P<0.01$ compared with Si-Ctl alone; \# $P<0.05$ compared with Si-Ctl treated with 8-Br-cAMP and MPA). (m) HESC were transfected with Si-Ctl or Si-CAPN7 at indicated concentration, followed by treatment with or without 8-Br-cAMP and MPA for an additional two or four days. PRL secreted into the medium were measured using ELIFA. $(n=3, * \star P<0.01$ compared with Si-Ctl alone; \# P $<0.05$ compared with Si-Ctl treated with 8-Br-cAMP and MPA). (n) The hormonal profile of pregnancy is simulated in ovariectomized mice, and decidualization is triggered by injection of sesame seed oil into the uterine lumen of one of the two uterine horns. The decidual response is supported by additional hormone treatments, and after 4 days the untreated and oil-injected uterine horns of each mouse are examined. The uterine weight ratio of the stimulated horns to control horns was examined, which were infected with Ad-LacZ or Ad-GFP-CAPN7. After artificial induction of decidualization, mouse uterine sections were stained with hematoxylin and eosin. Bar $=1000 \mu \mathrm{m}$. 


\section{CAPN7 suppresses the mRNA expressions of FoxO1's target genes}

a b
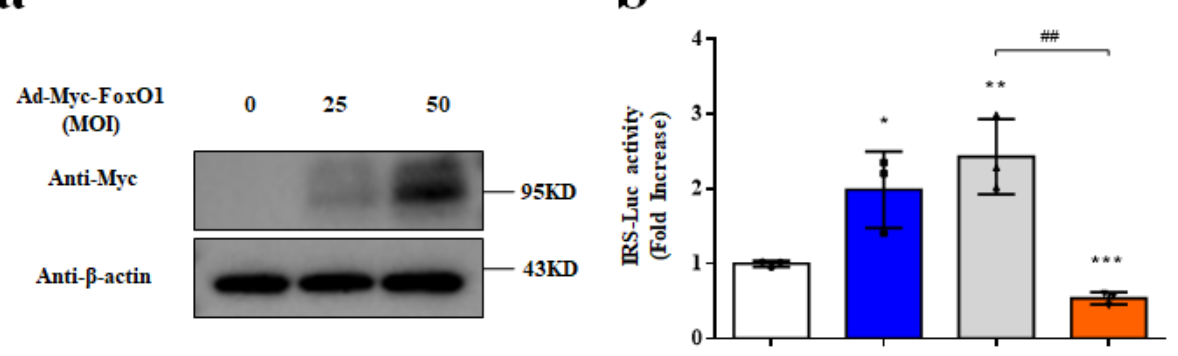

c

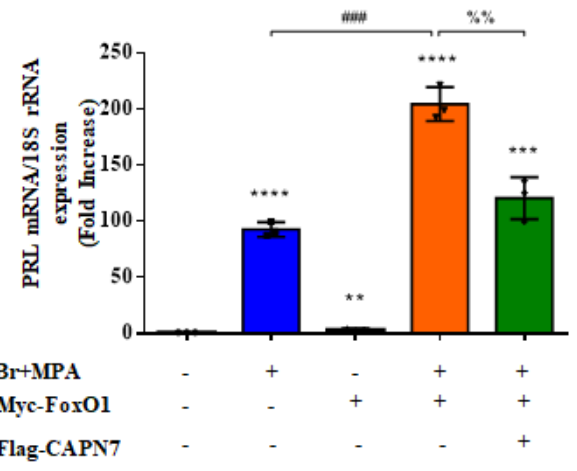

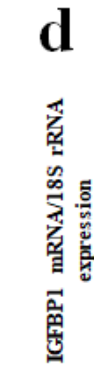

$8 \mathrm{Br}+\mathrm{MPA}$ Ad-Myc-FoxOl Ad-Flag-CAPN7 e

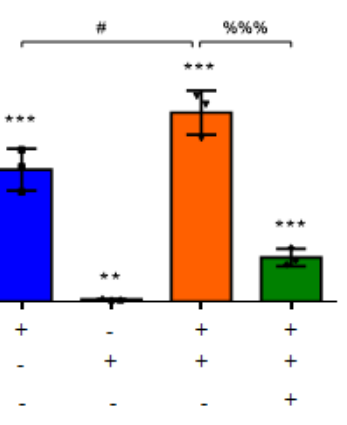

$8 \mathrm{Br}+\mathrm{MPA}$ Ad-Myc-FoxOl Ad-Flag-CAPN7

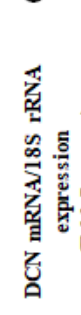

SBr+MPA Ad-Myc-F oxOl Ad-Flag-CAPN7 f

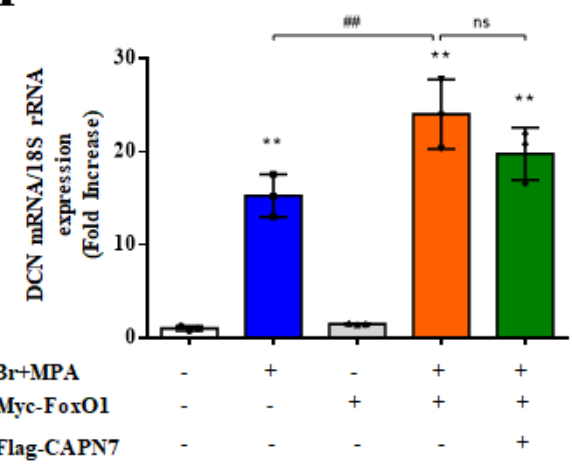

Figure 3

CAPN7 suppresses the mRNA expressions of FoxO1's target genes. (a) HESC were infected with Ad-LacZ or Ad-Myc-FoxO1 at the $\mathrm{MOI}$ indicated. Myc-FoxO1 protein expression was measured using Western blot.

(b) HESC were infected with Ad-LacZ or Ad-Flag-CAPN7 and/or Ad-Myc-Foxo1 at the MOI indicated for 24

h. Cells were then transfected with the IRS-Luc reporter construct using Lipo2000 transfection reagent, followed by treatment with or without 8-Br-cAMP and MPA. After $48 \mathrm{~h}$, luciferase assay was performed and normalized by constitutive Renilla luciferase. $(n=3, * p<0.05, * \star p<0.01$, $* \star \star p<0.001$, \#\#p<0.01). (c), (d), (e), (f) HESC were infected with Ad-LacZ, Ad-Flag-CAPN7 or Ad-Myc-Fox01 at the MOI indicated then treated with 8-Br-CAMP plus MPA for two days, after which PRL, IGFBP-1, DCN and TIMP-3 mRNA levels were separately measured using real-time PCR. $(n=3, * p<0.05, * \star p<0.01, * \star * p<0.001, * \star \star \star p<$ $0.0001, \# p<0.05, \# \# p<0.01, \# \# \#<<0.001, \% p<0.05, \% \% p<0.01)$. 


\section{CAPN7 promotes FoxO1's nuclear exclusion in decidualized HESC}

$\mathbf{a}$
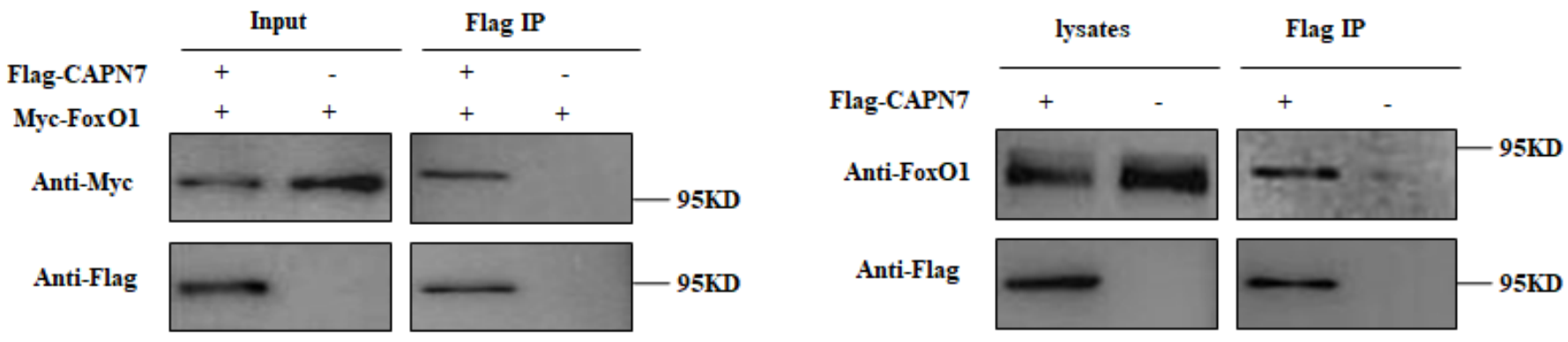

b
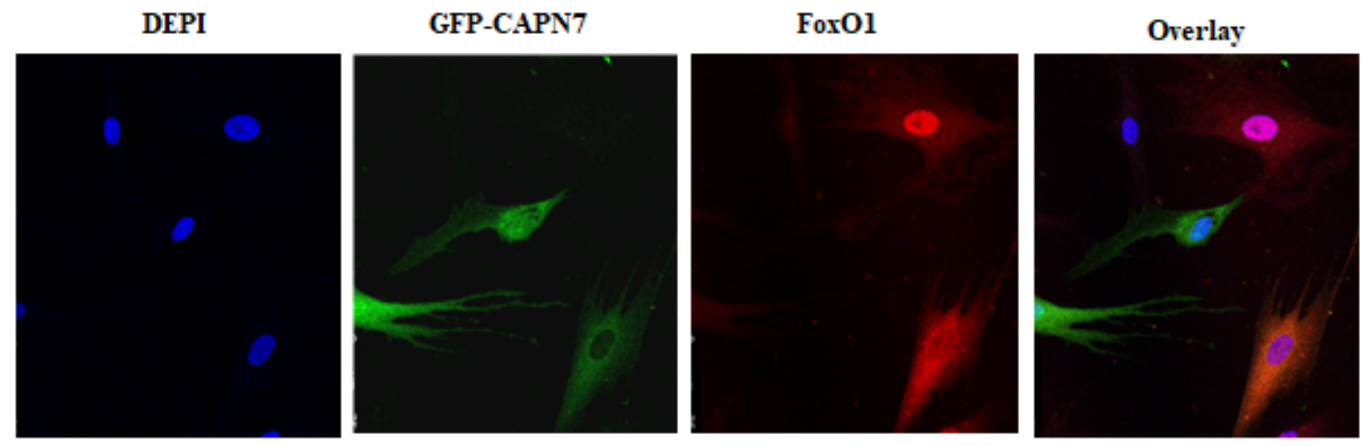

C
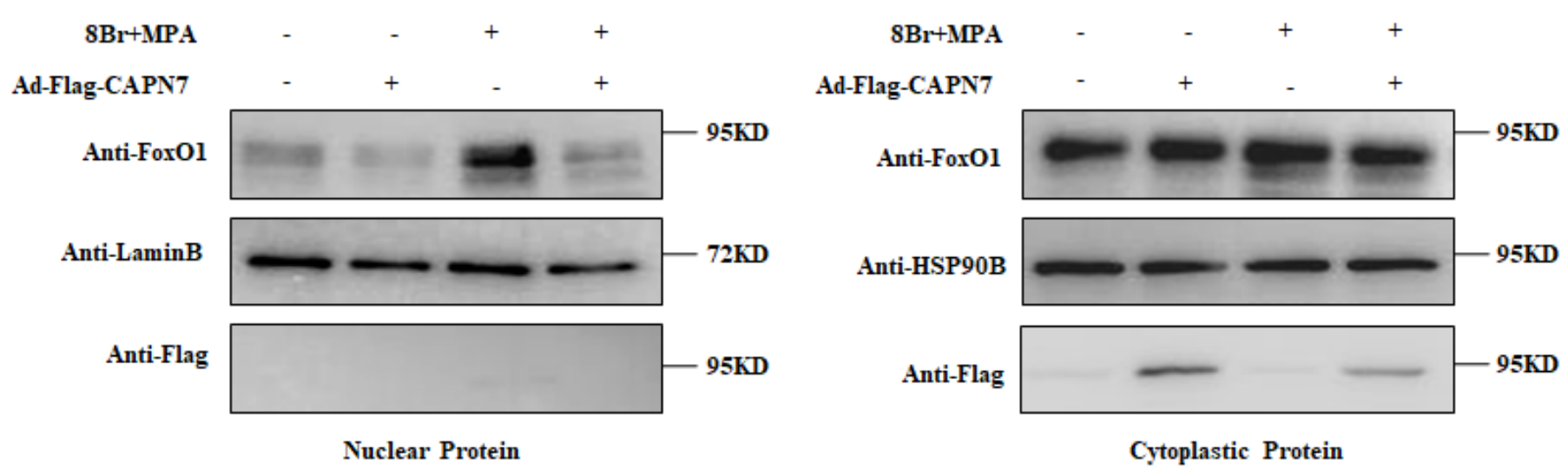

Figure 4

CAPN7 promotes Fox01's nuclear exclusion in decidualized HESC. (a) Immunoblot analysis of whole-cell lysates and anti-Myc immunoprecipitates of HEK293T cells transfected with Myc-Foxo1 alone or together with Flag-CAPN7 (left). Immunoblot analysis of whole-cell lysates and anti-Foxo1 immunoprecipitates of HESC infected with or without Ad-Flag-CAPN7, followed by treatment of 8-Br-CAMP and MPA for two days (right). (b) Immunofluorescence using FoxO1 antibody and Alexa Fluor 594-conjugated antibody to label the endogenous FoxO1 in HESC infected with Ad-GFP-CAPN7 with the treatment of 8-Br-cAMP and MPA for 60 hours. (c) Nuclear and cytoplasmic protein extract assay and Western Blot determining the FoxO1 
protein in HESC infected with or without Ad-Flag-CAPN7, with or without treatment of 8-Br-cAMP and MPA for 48 hours.

\section{CAPN7 hydrolyzes AKT1 and enhances the phosphorylation of FoxO1 and AKT1 in decidualized HESC}

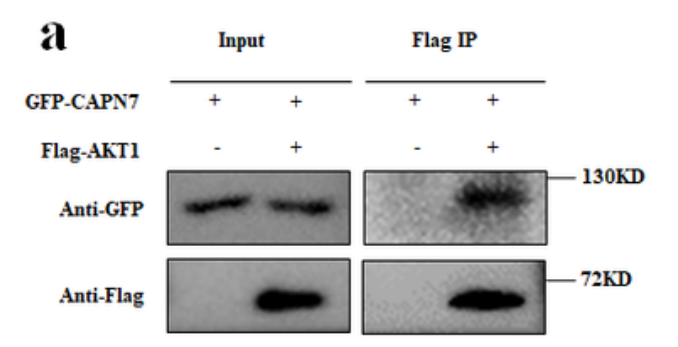

b

AKTl Domains
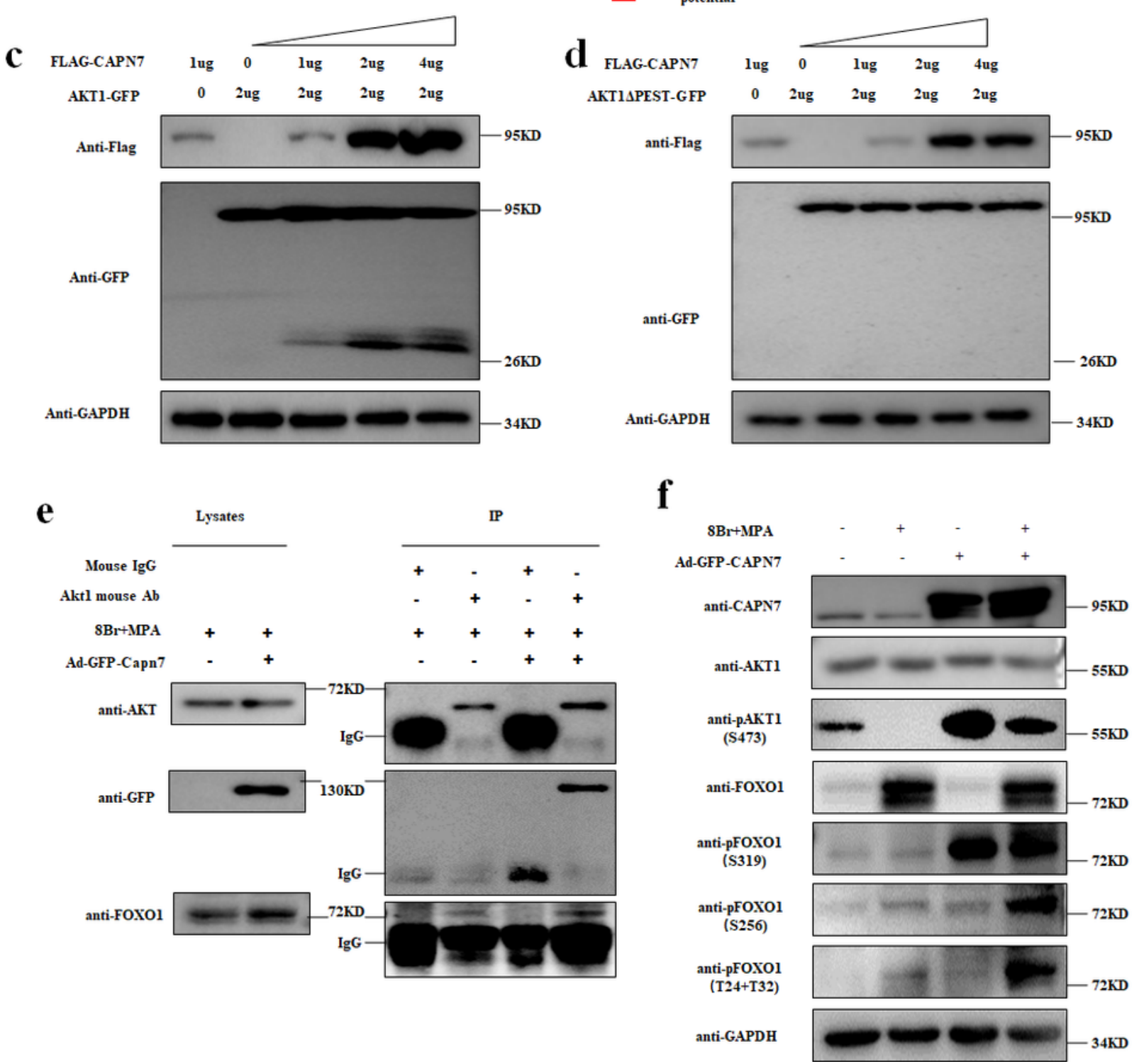

\section{Figure 5}

CAPN7 hydrolyzes AKT1 and enhances the phosphorylation of Fox01 and AKT1 in decidualized HESC.

(a) Immunoblot analysis of whole-cell lysates and anti-GFP immunoprecipitates of HEK293T cells transfected with GFP-CAPN7 alone or together with Flag-AKT1. (b) Schematic model of AKT1 containing 
the PEST sequence. (c) The HEK293T cells were transduced with Ad-Flag-CAPN7 and AKT1-GFP separately at indicated concentrations. Whole-cell lysates were analyzed by western blot analysis with the indicated antibodies. (d) The HEK293T cells were transduced with Ad-Flag-CAPN7 and AKT1 $\triangle$ PEST-GFP separately at indicated concentrations. Whole-cell lysates were analyzed by western blot analysis with the indicated antibodies. (e) Immunoblot analysis of whole-cell lysates and anti-FoxO1 immunoprecipitates of decidualized HESC transfected with GFP-CAPN7 or not. (f) The activity of AKT1/Fox01 pathway was assessed by Western blot in HESC infected with or without Ad-GFP-CAPN7, with or without the treatment of 8-Br-cAMP and MPA for 48 hours.

\section{Expressions of CAPN7, p-AKT1 and p-FoxO1 are increased in the EMs endometria}
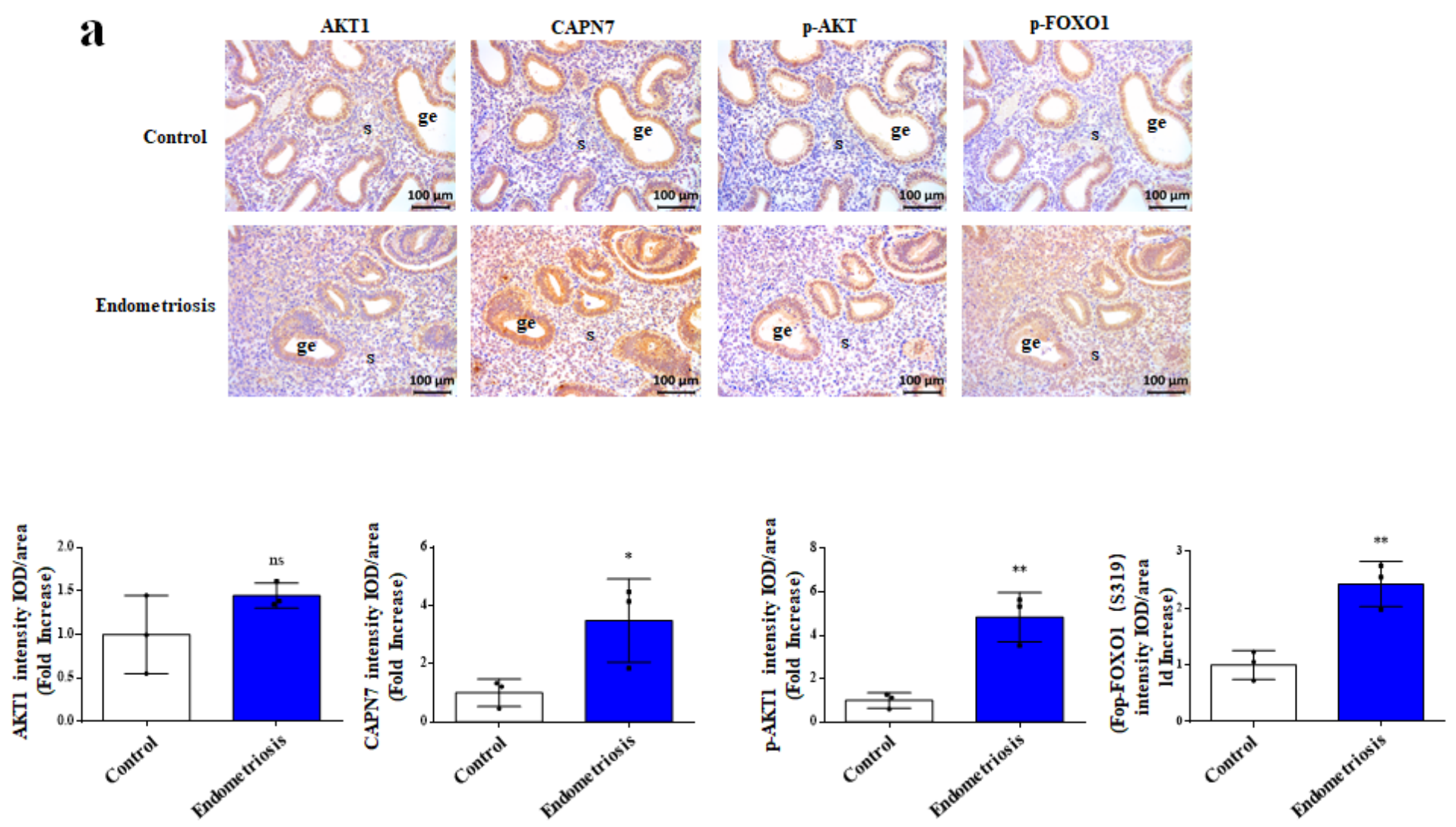

Figure 6

Expressions of CAPN7, p-AKT1 and p-FoxO1 are increased in the EMs endometria. (a) Immunohistochemical analysis was performed using AKT1, CAPN7, p-AKT1 and p-FoxO1 antibody, separately. Endometrial tissue samples from fertile women and patients with endometriosis are shown at $200 \times$ and $400 \times$ magnification. ${ }^{*} p<0.05$ compared with the control group; $* \star p<0.01$ compared with the control group . Scale bars: $100 \mu \mathrm{m}$. s, stroma; ge, glandular epithelium.

\section{Supplementary Files}

This is a list of supplementary files associated with this preprint. Click to download. 
- TableS1.docx

- Tables2.docx 